\title{
OFDM-System Design Using Adaptive Modulation and Channel Estimation for Underwater Acoustic Communication
}

\author{
Ravi Kumar $\mathrm{M} \mathrm{G}^{1}$ and Mrinal Sarvagya ${ }^{2}$ \\ ${ }^{1}$ Research Assistant, Dept. of ECE, REVA University, Bengaluru, Karnataka, \\ India \\ ${ }^{2}$ Professor, Dept. of ECE, REVA University, Bengaluru, Karnataka, India-560064 \\ *e-mail: ravimg9591@gmail.com and mrinalsarvagya@gmail.com
}

\begin{abstract}
Underwater Acoustic (UWA) channels are known as some of the most challenging communication media. Since the Underwater acoustic channels are time varying multipath channel achieving the high data rate, a low latency and a high throughput which often is a very difficult objective. In this work, we designed an OFDM transceiver system which is suitable for underwater acoustic communication with limited bandwidth to enhance the data rate at the receiver. To enhance the data rate at the OFDM receiver we have used efficient adaptive modulation scheme, channel estimation and channel equalization schemes for UWA communication. We have focused on the efficient modulation schemes like QPSK, DPSK and 16-QAM which is best suited for adaptive techniques based on the SNR of the Rayleigh fading channel to enhance the data rate. The simulation results show that the performance of the OFDM system with the use of Adaptive modulation is better when compared to the OFDM system without Adaptive modulation schemes. Also we have used the Kalman filter for channel estimation which minimizes the mean squared error and gives us the best estimation even in the presence of noise. From the simulation results, we have found that these modulation techniques and channel estimation method are best suited to achieve high data rate for underwater acoustic communication.
\end{abstract}

Keywords: UWA, OFDM, Adaptive modulation, Kalman filter, channel estimation, Channel equalization.

\section{Introduction}

In wireless communication, Underwater Acoustic (UWA) channels are known as some of the most challenging communication media. Compare to the electromagnetic wave channel and optical wave channel the UWA channel is considered by large transmission loss and delay, multi-path effect and Doppler Effect. Such parameters highly affect the performance of UWA communication and limits the channel capacity. In such a communication channel achieving high data rate, a low latency and a high throughput which often is a very challenging task [22].

The increasing need for high data rate in digital communications offers the Orthogonal Frequency-Division Multiplexing (OFDM) for achieving better performance in high rate data transmission [1]. OFDM is also a multicarrier modulation technique and considered as a best technique to combat frequency-selective fading channels in wireless communication systems [2,3]. OFDM is used in many applications like Digital Audio Broadcasting, Digital television(terrestrial), Wireless LAN IEEE 802.11a, IEEE 802.11g, IEEE 802.11n, IEEE 802.11ac, and IEEE 802.11ad, 4G and pre-4G cellular networks and mobile broadband standards. OFDM has high spectral efficiency as compared to other double sideband modulation schemes, spread spectrum, etc. and 
OFDM can easily adapt to severe channel conditions without complex time-domain equalization.

One of the most effective way for reliable communication is adaptive modulation that greatly enhances the utilization ratio of bandwidth [22]. The switching of modulation scheme is dependent on the UWA channel parameters as well as the SNR of the channel. The increasing demand for high bit rate digital communications makes efficient channel estimation and equalization techniques very attractive [1]. In this work the Kalman filter is used for effective channel estimation. The Kalman filter is an effective tool in several aspects, it supports estimation of past, present and future states and it can do even when the precise nature of the modelled system is unknown.

\section{Proposed Work}

Since Underwater Acoustic communication channel is a time-varying multipath, thus in such a communication channel achieving high data rate, a low latency and a high throughput which often is a very challenging task. By using the different techniques, the data rate of the OFDM for Underwater Acoustic communication can be increased. In this work, concentrating more on the efficient adaptive modulation techniques, Channel estimation and Equalization techniques. There are so many digital modulation techniques and channel estimation techniques which are well established in the communication field, choice of a suitable modulation technique and channel estimation/ equalization technique for an application depends on many parameters such as data rate, bit error rate and design complexity etc. This work includes that enhancing the data rate of the OFDM receiver by using Adaptive modulation and Kalman filter based Channel estimation schemes.

\section{System Design and Methodology}

OFDM is considered as one of the special types of multi-carrier modulation scheme which transmit by multiple sub carriers do not have similar frequencies and they are orthogonal to each other. Figure (1) shows the proposed OFDM system for UWA communication considered in this work.

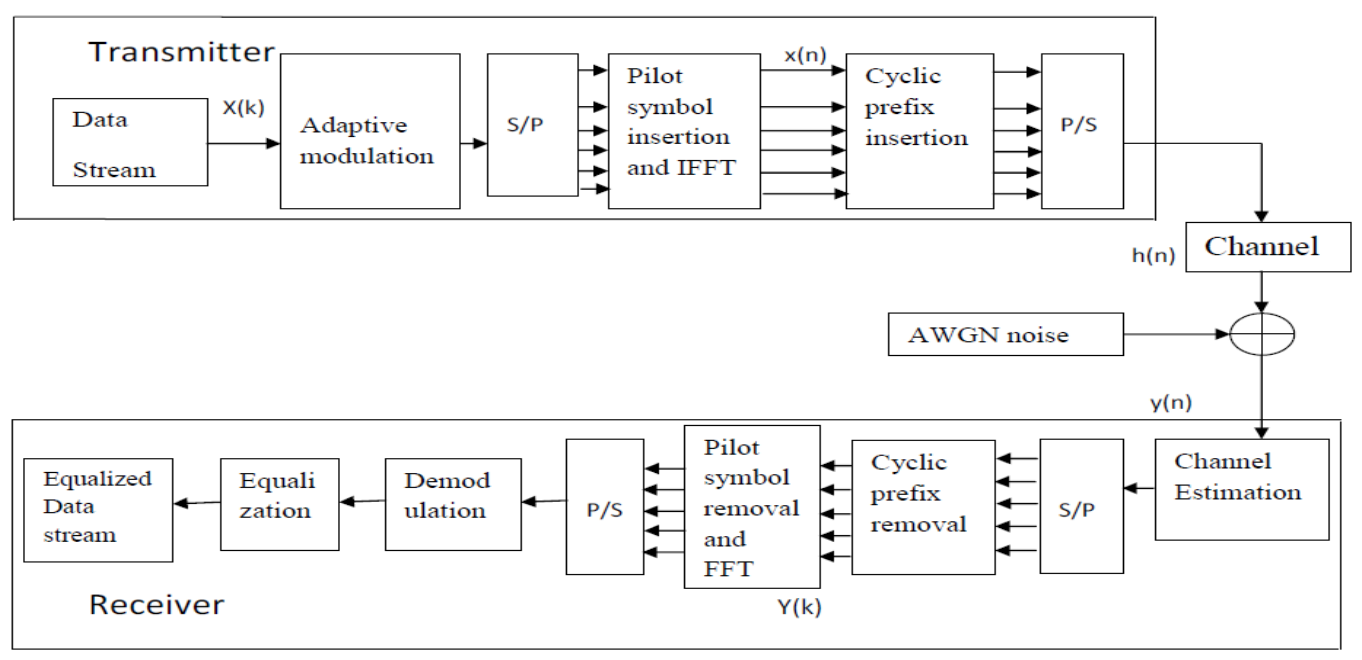

Figure 1. Proposed OFDM Transceiver System

First the data stream is generated which is in binary form. The random binary signal models the raw information which would be transmitted and then the signal fed into transmitter [11]. After fed into the transmitter the transmitted signal is modulated using the modulation techniques. In this paper we establish some efficient adaptive modulation 
schemes such as QPSK, DPSK and 16-QAM for underwater acoustic communication in order to get high data rates by considering ' $\mathrm{N}$ ' subcarriers (256 subcarriers). In this work, we experimented and analyzed individual modulation scheme results with OFDM for underwater communication. Initially we assumed the Eb/No ratio from 1 to 30 and using the $\mathrm{Eb} / \mathrm{No}$ values we calculated the SNR values initially. Thus we done the OFDM system to switch the appropriate modulation scheme which depends upon on the SNR range of the channel. The modulated serial data stream is the converted into parallel data. A pilot signal which is generally of single frequency, which is transmitted over a communications system for control, supervisory, equalization and reference purpose[12]. In this experiment we insert the pilot symbols of length 4 for each frame in the transmitter side and after FFT algorithm we remove the pilot symbols at the receiver side. The capability to generate and to demodulate the signal with the use of FFT algorithm is one of the main factors for OFDM to become popular in transmission schemes [13]. By using IFFT/FFT techniques, implementation of modulation and demodulation is computationally efficient. The FFT and IFFT algorithms can be obtained by the equations (1) and (2) respectively.

$$
\begin{aligned}
& X_{0}(k)=\operatorname{FFT}\left[x_{0}(n)\right]=\frac{1}{N} \sum_{k=0}^{N-1} \mathrm{x}_{0}(n) e^{j 2 \pi k n} \\
& \mathrm{x}_{p}(n)=\operatorname{IFFT}\left[X_{0}(k)\right]=\frac{1}{N} \sum_{k=0}^{N-1} X_{0}(k) e^{j 2 \pi k n}
\end{aligned}
$$

Cyclic prefix is the parameter that enables the OFDM signal to operate reliably and it is used to defend the OFDM signals from inter symbol interference. In this experiment we used acyclic prefix of length 16 to avoid the OFDM signals from inter symbol interference [12]. The received signal can be given by,

$$
y_{k}(n)=h_{k}(n) * x_{k}(n)+z(n)
$$

The channel coefficients vector $h_{k}(n)=\left[h_{k}(n, 0), h_{k}(n, 1) \ldots \ldots h_{k}(n, L-1)\right]$, where $h_{k}(n, l)$ is the $l^{t h}$ tap at $n^{\text {th }}$ time instant. The state vector is obtained as $x_{k}(n)=$ $\left[x_{0}, x_{1}, x_{2} \ldots \ldots x_{k}\right]^{T}$.

In this experiment we considered the Rayleigh fading channel as the media for communication [2]. The Doppler spread, noise, high path loss, multipath and variable propagation delay affect the underwater acoustic communications severely. The collective effect of this phenomenon causes the UWA channel to be temporally and spatially variable. This limits the available bandwidth and makes it more dependent on both range and frequency. The parameters that affect UWA communication are (a). Transmission loss which occurs due to two factors: attenuation and geometric spreading loss, (b). Acoustic Noise occurred is mainly caused by shipping activities and machinery noise, (c). Attenuation which can be generally attributed to absorption, due to the translation of energy of the propagating acoustic wave into heat. The SNR can be calculated for the channel based on the Eb/No ratio.

\subsection{Kalman Filter based Channel Estimation}

Channel estimation is a scheme, in which the channel state information is retrieved by using the channel impulse response. Kalman filtering is an efficient technique to remove impurities in linear systems [8]. The Kalman filter is a recursive filter that is with the help of state space methods and recursive algorithms [12]. The Kalman filter algorithm includes two steps; a) Prediction - It refers to projecting forward the current state to gain the estimates for next step. It is therefore known as time update step. b) Correction - It is the feedback stage that incorporates new measurements into the estimates. It is therefore known as measurement step. This process is repeated for each state with value from previous state as initial value. Therefore, Kalman filter is called a recursive filter and it is represented in state-space model and the state-space model consists of two equations:

$$
\mathrm{x}_{\mathrm{k}}=\mathrm{Ax}_{\mathrm{k}-1}+\mathrm{w}_{\mathrm{k}-1}
$$




$$
\mathrm{y}_{\mathrm{k}}=\mathrm{Cx}_{\mathrm{k}}+\mathrm{v}_{\mathrm{k}}
$$

$\mathrm{x}_{\mathrm{k}}$ is the $\mathrm{N}$-dimensional state vector at $\mathrm{k}=1,2,3, \ldots \mathrm{n}$. Process noise ' $\mathrm{w}$ ' is the $\mathrm{N}$ dimensional uncorrelated input vector of state equation. $\mathrm{y}_{\mathrm{k}}$ is the M-dimensional noisy observation vector and the measurement noise $\mathrm{v}_{\mathrm{k}}$ is a zero mean white Gaussian noise. ' $A$ ' is the state transition matrix which can be obtained using the Yule-Walker equations (AR process) and ' $\mathrm{C}$ ' is the output matrix which are given by

$$
\mathrm{A}=\left[\begin{array}{ccc}
-\mathrm{A} 1 & -\mathrm{A} 2 & \ldots \ldots . \mathrm{A}(\mathrm{p}) \\
\mathrm{I} & 0 & \ldots \ldots \ldots \ldots .0 \\
0 & \mathrm{I} & \ldots \ldots \ldots \ldots .
\end{array}\right]
$$

and $\mathrm{C}=[\mathrm{I}, 0, \ldots .0]$. Where $\mathrm{I}$ is the $\mathrm{N} \times \mathrm{N}$ identity matrix and $\mathrm{A} 1, \mathrm{~A} 2 \ldots . . \mathrm{A}(\mathrm{p})$ are the $\mathrm{AR}$ model coefficients. At the $\mathrm{k}-1^{\text {th }}$ iteration, the state vector is considered as zero vectors and the state transition matrix is calculated using the Yule Walker AR process. The Kalman filter is then applied to estimate the unknown state of the system. Received signal $y_{k}(n)$ at each instant is given as input observation to Kalman filter algorithm [13] and the following figure (2) shows the Kalman filter algorithm. There is a consideration of two ideal equations: Time Update and Measurement Update and both set of e equations are applied at each $\mathrm{k}^{\text {th }}$ state.

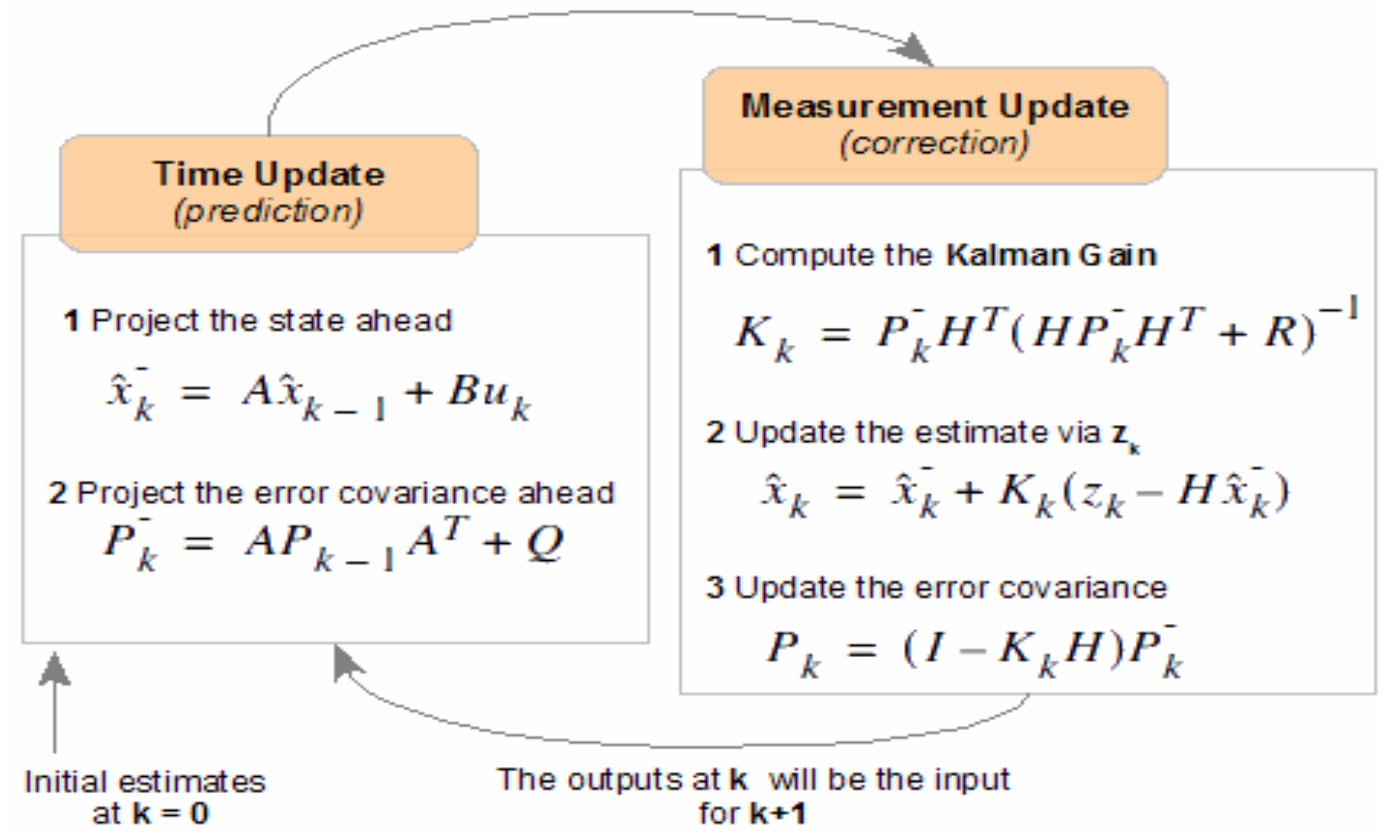

Figure 2. Kalman Filter Algorithm

Where,

$\hat{\mathrm{x}}$ :Estimation of the state vector

$\hat{\mathrm{x}}^{-}$:Prediction of the state vector

$\mathrm{z}$ :Measurement values vector

$\mathrm{K}:$ Kalman gain

$\overline{\mathrm{P}}$ :Prediction of the error covariance

$\mathrm{P}:$ :Error covariance update

$\mathrm{I}$ :Unit matrix

Q and R : Covariance matrices of system noise and measurement noise respectively. 


\section{Simulation Results and Discussion}

\subsection{Simulation Parameters}

In this work, we have assumed some OFDM parameters for simulation. The state transition matrix is calculated using Yule-Walker AR process. Both Process noise and measurement noise is assumed to be Gaussian noise. All the OFDM parameters are tabulated in table (1). The channel under consideration is Rayleigh fading channel.

Table 1. OFDM Parameters

\begin{tabular}{|l|l|}
\hline No of subcarriers & 512 \\
\hline Cyclic prefix length & 16 \\
\hline Sampling period of channel & $1 \mathrm{e}^{-3}$ \\
\hline Max Doppler frequency shift & 0 \\
\hline Distance & $15 \mathrm{~m}$ \\
\hline No of OFDM symbols & 1000 \\
\hline FFT size & 256 \\
\hline Carrier frequency & $8 \mathrm{KHz}$ \\
\hline Receiver noise bandwidth & $2 \mathrm{KHz}$ \\
\hline No of pilot symbols & 4 \\
\hline Modulation schemes & QPSK, DPSK, 16-QAM \\
\hline
\end{tabular}

\subsection{Algorithm Overview}

The overall algorithm for OFDM system works as follows:

1. Initialize the variables and matrices.

2. Generate the random input serial data stream.

3. Modulate the data using efficient modulation techniques.

4. Convert the serial data into parallel data and insert the pilot symbols then apply IFFT algorithm.

5. Insert the cyclic prefix symbols to avoid Inter symbol interference.

6. Convert the parallel data into serial data and pass through the Rayleigh channel in which AWGN noise is added.

7. Convert the serial data into parallel data and remove the cyclic prefix symbols.

8. Perform Channel estimation using Kalman filter.

9. Apply FFT algorithm and remove the pilot symbols.

10. Convert the parallel data into serial data and demodulate the data.

11. Equalize the modulated data using equalizer.

12. Calculate the SNR, BER and plot the results.

13. Based on the SNR the modulation techniques will be selected.

In this work, we focus on finding the efficient adaptive modulation techniques, channel estimation technique and channel equalization schemes which are suitable for the underwater environment even in the presence of AWGN noise and channel noise. In this experiment, we have considered efficient modulation schemes like QPSK, DPSK and 16QAM and verified the BER performance individually. These schemes typically give a better performance compared to other modulation techniques. Thus by applying the adaptive method in the modulation process the BER or SNR performance were optimized and the efficient data rate was achieved. From the simulation results also found that the Kalman filter is suitable for signal tracking using channel estimation compared to the other channel estimation techniques for UWA communication. The simulation results of 
the BER performance of DPSK, QPSK, 16-QAM modulation techniques are shown in figure (3) to figure (5) respectively and the BER performance of the OFDM system with adaptive modulation is shown in figure (6). The simulation of the comparison of the real states and the estimated states is shown in the figure (7) and the results for signal tracking using Kalman filter in AWGN channel and Rayleigh channel are shown in figure (8) to figure (9) respectively. Table (2) shows the BER performance without Adaptive modulation schemes for the Eb/No ratio of 1-30 dB:

Table 2. BER Performance of different Modulation Schemes

\begin{tabular}{|c|c|}
\hline Modulation schemes & BER \\
\hline QAM & 0.0645 \\
\hline DPSK & 0.0501 \\
\hline QPSK & 0.0656 \\
\hline
\end{tabular}

After performing the Adaptive modulation we can achieve the BER of $\mathbf{0 . 0 4 9 2}$. This simulation results proves that the Adaptive or switching of appropriate modulation schemes based on the SNR will improve the performance of the OFDM system.

\subsection{Simulation Results}

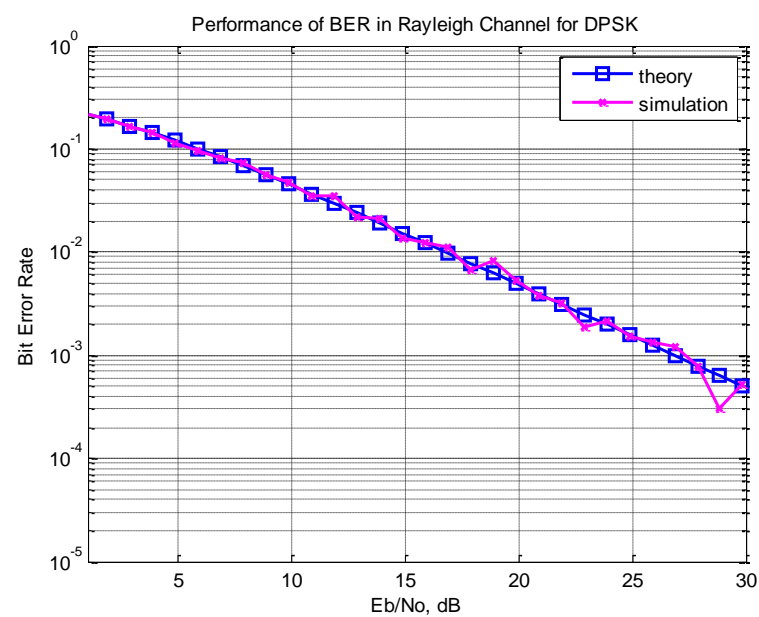

Figure 3. BER Performance in Rayleigh Channel for DPSK 


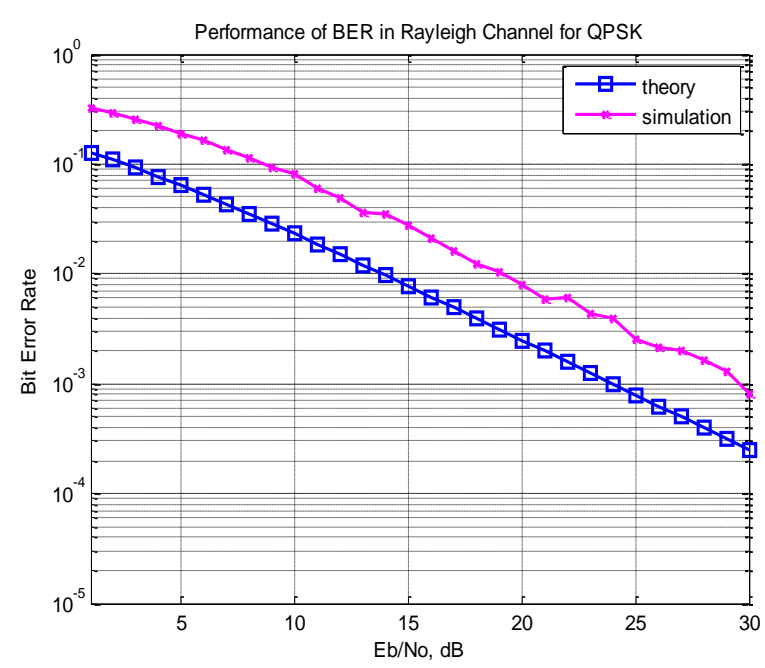

Figure 4. BER Performance in Rayleigh Channel for QPSK

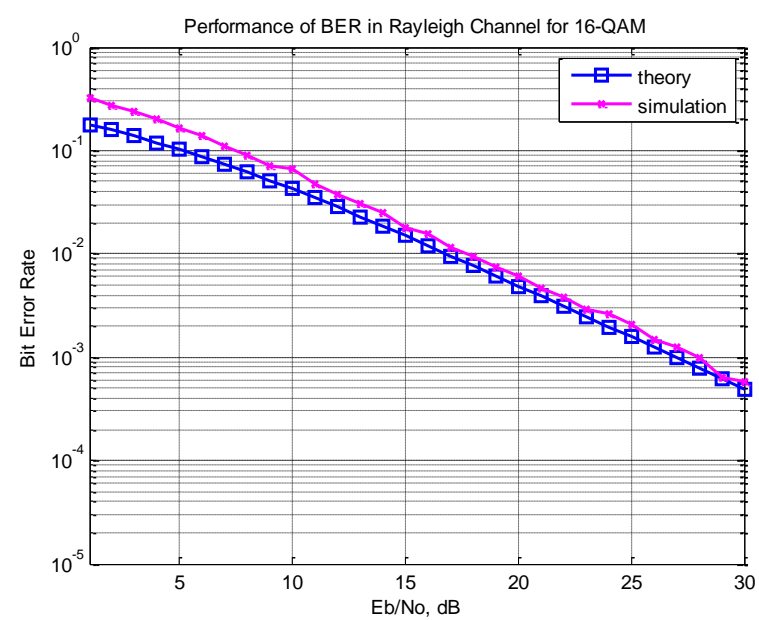

Figure 5. BER Performance in Rayleigh Channel for 16-QAM

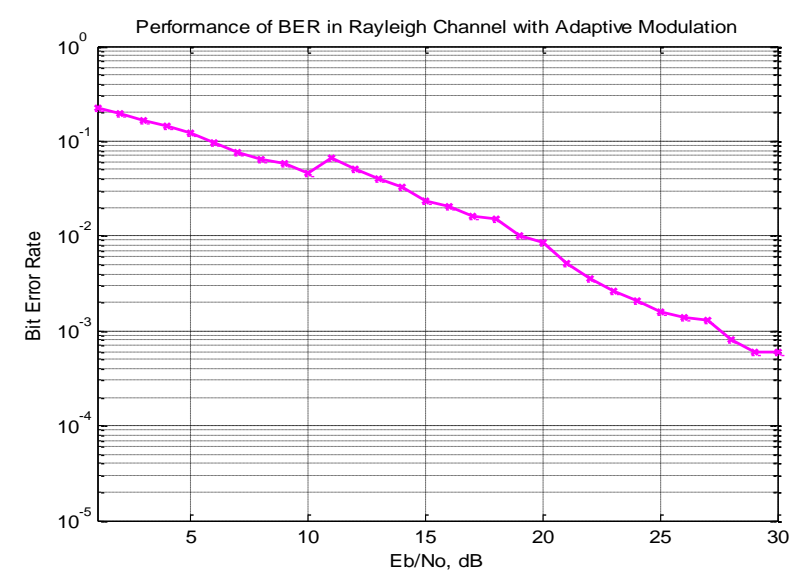

Figure 6. Performance of BER in Rayleigh Channel with Adaptive Modulation 


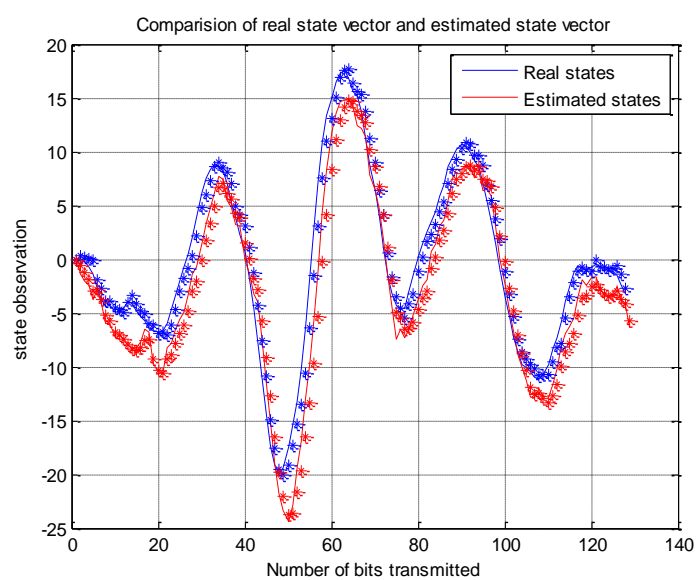

Figure 7. Comparision of Real State Vector and Estimated State Vector

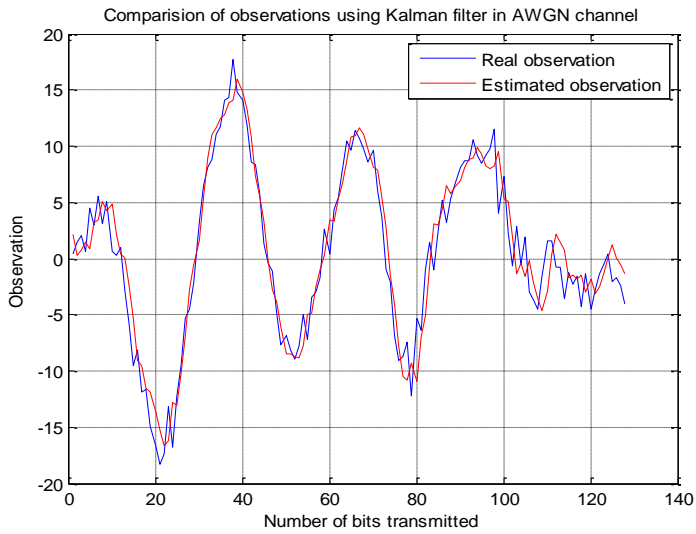

Figure 8. Comparison of Real Observation and Estimated Observation in AWGN Channel using Kalman Filter

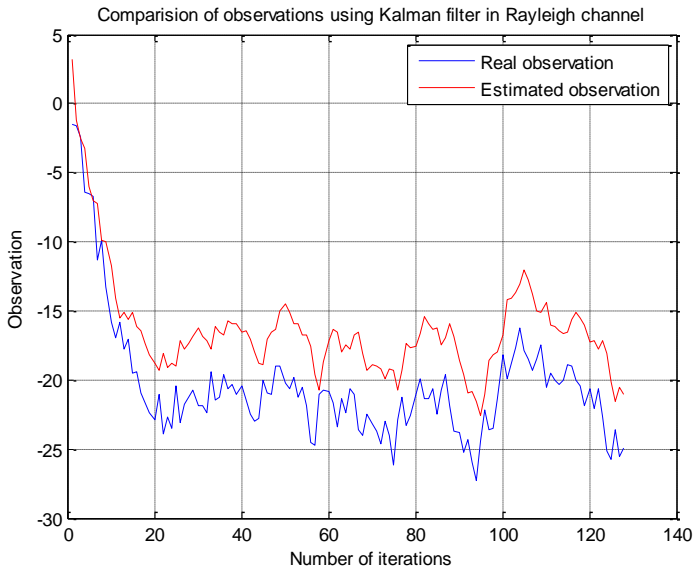

Figure 9. Comparison of Observations using Kalman Filter in Rayleigh channel 


\section{Conclusion and Future Work}

Underwater Acoustic (UWA) Communication is some of the challenging and interesting area of research in nowadays. Since underwater acoustic channel is highly limited in bandwidth achieving high data rate, low latency and high throughput is a very challenging task. To achieve these objectives, we have used many techniques that include efficient Adaptive modulation scheme, Channel estimation using Kalman filter and channel equalization schemes with OFDM multicarrier modulation technique. From the simulation results we found that some modulation schemes like QPSK, DPSK and 16QAM are the best adaptive modulation schemes for underwater communication for highly limited bandwidth even in the presence of channel noise. The simulation results show that the performance of the OFDM system with the use of Adaptive modulation is better when compared to the OFDM system without Adaptive modulation schemes. The Kalman filter is used for channel estimation and the signal tracking is achieved efficiently by using the Kalman filter. Thus by using the adaptive modulation schemes based on the SNR of the Rayleigh channel, channel estimation techniques like Kalman filter and channel equalization techniques like LMS, MMSE, we designed OFDM receiver which has high data rate for underwater communication. In future we can design the MIMO design which will have high data rate in the receiver by using other ways such as use of channel coding techniques and adaptive channel estimation schemes, etc. We can design the receiver and verify the performance using numerical simulation and Mathematical model.

\section{Acknowledgements}

The authors wish to thank Navel Research Board (NRB), Delhi, INDIA, for funding the part of the project under NRB Research Scheme, Grant No. NRB/4003/PG/365 Dated 21 September 2015.

\section{References}

[1] A. Zaier and R. Bouallègue, "A full performance analysis of channel estimation methods for time varying ofdm systems", International Journal of Mobile Network Communications \& Telematics (IJMNCT), vol. 1, no. 2, (2011).

[2] B. Li, J. Huang, S. L. Zhou, K. Ball and M. Janovic, "MIMO-OFDM for high rate underwater acoustic Communications", IEEE Journal of Oceanic Engineering, (2009).

[3] T. Melodia, H. S. H. Jian, L. Chungkuo and E. Demirors, "Advances in Underwater Acoustic Networking", Chapter 23, First Edition. By TommasoMelodia, Copyright, (2012), John Wiley \& Sons, Inc.

[4] I. F Akyildiz, T. Melodia, and K. R. Chowdhury, "A Survey on Wireless Multimedia Sensor Networks", Computer Networks (Elsevier), vol. 51, no. 4, (2007), pp. 921-960.

[5] M. U. Cella, R. Johnstone and N. Shuley, "Electromagnetic wave wireless communication in shallow water coastal environment: Theoretical analysis and experimental results", In ACM International Workshop on UnderWater Networks (WUWNet'09), Berkeley, California, USA, (2009), pp. 9:1-9:8.

[6] N. Farr, A. Bowen, J. Ware, C. Pontbriand and M. Tivey, "An integrated, underwater optical/acoustic communications system", In Proceedings of IEEE OCEANS, (2010), pp. 1-6.

[7] I. F. Akyildiz, D. Pompili and T. Melodia, "Challenges for Efficient Communication in Underwater Acoustic Sensor Networks", ACM Sigbed Review, (2004).

[8] N. Valdaya and M. Kumawat, "A Novel Algorithm for Efficient channel estimation using MMSEKalman estimator and Median filtering", International journal of computer applications(0975-8887), vol. 100, no. 13, (2014).

[9] A. Kapoor and I. Khurana, "Channel Estimation based on Kalman filtering with BER reduction in MIMO-OFDM syatems", International journal of computer applications (0975-8887), vol. 97, no. 1, (2014).

[10] J. Faezah and K. Sabira, "Adaptive Modulation for OFDM Systems", International Journal of Communication Networks and Information Security (IJCNIS), vol. 1, no. 2, (2009).

[11] C. F. Lin, W. C. Wu and J. C. Y. Lee, "Underwater Acoustic Multimedia Communication Based on MIMO-OFDM", Wireless PersCommun, vol. 71, (2013), pp.1231-1245.

[12] M. Gupta, R. Nema and R. S. Mishra, "Bit error rate performance in OFDM system using MMSE and MLSE Equalizer over Rayleigh fading channel through the BPSK, QPSK, 4QAM and 16 QAM Modulation technique”, IJERA, ISSN:2248-9622, vol. 1, iss. 3, pp. 1005-1011 
[13] K. P. Bagadi and S. Das, "MIMO-OFDM Channel Estimation using Pilot Carries", International Journal of Computer Applications (0975 - 8887), vol. 2, no. 3, (2010).

[14] M. Oltean, "An Introduction to Orthogonal Frequency Division Multiplexing", Universitatea "Politehnica" Timişoara.

[15] R. Jain, "Kalman filter based Channel Estimation", International journal of Research and technology (IJERT), ISSN: 2278-0181, vol. 3, iss. 4, (2014).

[16] L. Freitag, M. Stojanovic, S. Singh and M. Johnson, "Analysis of channel effects on direct-sequence and frequency-hopped spread spectrum acoustic communications", IEEE Journal of Oceanic Engineering, vol. 26, no. 4, (2001), pp. 586-593, 2001.

[17] N. K. Noordin, B. M. Ali, N. Ismail and S. S. Jamuar, "Adaptive techniques in Orthogonal frequency division Multiplexing in mobile radio Environment", International Journal of Engineering and technology, vol. 1, no. 2, (2004), pp. 115-123.

[18] M. D. G. Sadeque, "Bit Error Rate (BER) Comparison of AWGN Channels for Different Type's Digital Modulation Using MATLAB Simulink", ASRJETS, ISSN (Print) 2313-4410, ISSN (Online) 2313-4402 (C) Global Society of Scientific Research and Researchers.

[19] B. K. Patel and J. Agarwal, "Comparative Study of Bit Error Rate with Channel Estimation in OFDM System for M-ary Different Modulation Techniques", International Journal of Computer Applications (0975 - 8887), vol. 95, no. 8, (2014).

[20] Z. L. Gopal, "Kalman Filtering for SNR Estimation in AWGN and Fading Channels", Proceedings of the 2009 IEEE $9^{\text {th }}$ International Conference on Communications, (2009).

[21] C. Komninakis, "Multi-output fading channel tracking and equalization using Kalman Estimation", IEEE transaction on Signal Processing, vol. 50, no. 5, (2002).

[22] T. J Apren, "Channel estimation with Extended Kalman filter for Fading channels", IJECCT, vol. 3, iss. $5,(\mathbf{2 0 1 3})$.

[23] S. Xiaohong, W. Haiyan, Z. Yuzhi and Z. Ruiqin, "Adaptive Technique for Underwater Acoustic Communication", chapter 4, Underwater Acoustics. 